\title{
Traveling Wave Solution in a Diffusive Predator-Prey System with Holling Type-IV Functional Response
}

\author{
Deniu Yang, ${ }^{1}$ Lihan Liu, ${ }^{2}$ and Hongyong Wang ${ }^{3}$ \\ ${ }^{1}$ Polytechnic Institute of Jiangxi Science and Technology Normal University, Nanchang 330038, China \\ ${ }^{2}$ Department of Mathematics, Chongqing Normal University, Chongqing 400030, China \\ ${ }^{3}$ School of Mathematics and Physics, University of South China, Hengyang 421001, China
}

Correspondence should be addressed to Hongyong Wang; whylj_love@126.com

Received 13 November 2013; Accepted 27 January 2014; Published 6 March 2014

Academic Editor: Chaudry Masood Khalique

Copyright (C) 2014 Deniu Yang et al. This is an open access article distributed under the Creative Commons Attribution License, which permits unrestricted use, distribution, and reproduction in any medium, provided the original work is properly cited.

We establish the existence of traveling wave solution for a reaction-diffusion predator-prey system with Holling type-IV functional response. For simplicity, only one space dimension will be involved, the traveling solution equivalent to the heteroclinic orbits in $R^{3}$. The methods used to prove the result are the shooting argument and the invariant manifold theory.

\section{Introduction}

The paper will study the traveling wave solution for a diffusive predator-prey system with Holling type-IV functional response, which is as follows:

$$
\begin{gathered}
u_{t}=d_{1} u_{x x}+A u\left(1-\frac{u}{K}\right)-\frac{B u w}{1+E u^{2}}, \\
w_{t}=d_{2} w_{x x}+w\left(\frac{D u}{1+E u^{2}}-C\right) .
\end{gathered}
$$

All parameters are positive constant. The functions $u(x, t)$ and $w(x, t)$ are the densities of the prey and predator, respectively; $d_{1}$ and $d_{2}$ are diffusive rates of the prey and predator, respectively; $K$ is the carrying capacity of the prey; $C$ is the death rate of the predator; and $A$ is the growth factor of the prey. We may refer to $[1,2]$ for more biological implications.

Recently, the system (1) and some related systems have been studied by many researchers for an understanding of the most basic features of a spatially distributed interaction; we can refer to [3-10]. Gardner [8] proved the existence of traveling wave solutions for a diffusive predator-prey system with Holling type-II functional response by using the connection index. Numerical simulation in Owen and Lewis [11] shows that a diffusive predator-prey system with
Holling type-II functional response, when the diffusive rates of the prey and the predator are not zero, possesses traveling wave solutions. Huang et al. [12] proved theoretically that the numerical simulation in [11] is true. Huang et al. considered the system

$$
\begin{aligned}
& u_{t}=u\left[\alpha(b-u)-\frac{w}{1+u}\right], \\
& w_{t}=w_{x x}-w\left(1-\frac{\beta u}{1+u}\right),
\end{aligned}
$$

and they obtained that if $c>\sqrt{4(b \beta-1-b) /(1+b)},(b+$ $1) / b<\beta<b /(b-1)$, and $(1-\alpha)(\beta-1) \geq(2 \beta /(1+b))$ $\sqrt{(b \beta-1-b) /(1+b)}$, then there are nonnegative solutions of system (2) satisfying $w(+\infty)=\alpha(1 /(\beta-1)+1)(b-1 /(\beta-1))$, $w(-\infty)=0, u(+\infty)=1 /(\beta-1), u(-\infty)=b$.

Dunbar [13] studied the following system:

$$
\begin{gathered}
U_{t}=U(1-W), \\
W_{t}=W_{x x}+\rho W(U-1)
\end{gathered}
$$

and obtained the following.

(a) If $0<c<\sqrt{4 \alpha(1-\beta)}$, then there exist traveling wave front solutions of the system (3) satisfying $u(-\infty)=0$, $w(-\infty)=0, u(+\infty)=\beta, w(+\infty)=1-\beta$. 
(b) If $c \geq \sqrt{4 \alpha(1-\beta)}$, then there exist traveling wave front solutions of the system (3) satisfying $u(-\infty)=1$, $w(-\infty)=0, u(+\infty)=\beta, w(+\infty)=1-\beta$.

Dunbar [14] investigated the system

$$
\begin{aligned}
& U_{t}=\alpha U(\gamma-U)-\frac{U W}{1+U}, \\
& W_{t}=W_{x x}-W+\beta \frac{U W}{1+U}
\end{aligned}
$$

and obtained that if $c^{2}>4(\gamma \beta-\gamma-1) /(1+\gamma)$ and $(1+$ $\gamma) / \gamma<\beta \leq \gamma /(\gamma-1)$, then there is a bounded solution of (4) satisfying $u(-\infty)=\gamma, w(-\infty)=0, u(+\infty)=1 /(\beta-1)$, and $w(+\infty)=\alpha(\gamma-1 /(\beta-1))(1+1 /(\beta-1))$.

$\mathrm{Li}$ and $\mathrm{Wu}[15]$ studied a system with Holling type-III functional response and proved the existence of traveling wave solutions by using the shooting argument in $R^{3}$ together with a Lyapunov function [16], LaSalle's invariance principle [17], and the Hopf bifurcation theorem [18]. We may refer to Murray [19], Mischaikow and Reineck [20], and Volpert et al. [21] for more results.

We notice that the Holling type-II and the Holling typeIII functional response are monotonic in the first quadrant, while the Holling type-IV functional response considered in this paper is nonmonotonic in the first quadrant. It is an interesting problem to know whether the above results are available for the system (1). We should mention that although the techniques used here are similar to those in $[12-15,22]$, there are several differences. Firstly, it is a more complex system. The systems studied in $[13,22]$ are the ones with the Lotka-Volterra functional response. The systems studied in $[12,14,15]$ are the ones with the Holling type-II or Holling type-III functional response. Secondly, we construct a different Wazewski set $W$ and a new Lyapunov function. For simplicity, we assume that $d_{1}=0$ can be considered to correspond to a situation in which the prey species is evenly distributed. We should mention that the assumption is not essential.

For further simplification, taking

$$
\begin{gathered}
u^{*}=\sqrt{E} u, \quad w^{*}=\frac{B}{C} w, \quad x^{\prime}=\sqrt{\frac{C}{d_{2}} x,} \\
t^{\prime}=C t, \quad \alpha=\frac{A}{\sqrt{E} C K}, \quad b=\sqrt{E} K, \quad \beta=\frac{D}{\sqrt{E} C},
\end{gathered}
$$

and dropping the stars on $u, w$ and the primes on $x, t$ for convenience, we obtain

$$
\begin{aligned}
& u_{t}=\alpha u(b-u)-\frac{u w}{1+u^{2}}, \\
& w_{t}=w_{x x}-w+\frac{\beta u w}{1+u^{2}}
\end{aligned}
$$

There are several reasonable parameter restrictions. We assume that $b>1$ or equivalently that $E>1 / K^{2}$, so that the satiation effect is great enough. We also assume that $\alpha>0$ and $\beta>2$, which ensure that the system (6) has positive equilibrium point corresponding to constant coexistence of the two species. Obviously, the system (6) has four equilibria points: $(0,0),(b, 0),\left(u_{0}, w_{0}\right)$, and $\left(u_{1}, w_{1}\right)$, which are equilibria of the corresponding ODE system without diffusion, where

$$
\begin{array}{ll}
u_{0}=\frac{\beta-\sqrt{\beta^{2}-4}}{2}, & w_{0}=\alpha\left(b-u_{0}\right)\left(1+u_{0}^{2}\right), \\
u_{1}=\frac{\beta+\sqrt{\beta^{2}-4}}{2}, & w_{1}=\alpha\left(b-u_{1}\right)\left(1+u_{1}^{2}\right) .
\end{array}
$$

In this paper, we also require that $b<u_{1}$, which ensures that equations (6) has only a positive equilibrium. We notice that $u_{0}<b$, so the system (6) has only one positive equilibrium point. The equilibrium $(0,0)$, representing the absence of both species, is a saddle point. The equilibrium $(b, 0)$, representing the population of the prey at the environmental carrying capacity in the absence of predators, is unstable. The equilibrium $\left(u_{0}, w_{0}\right)$, representing the time constant coexistence of both species, is stable. We establish the traveling wave solution connecting the equilibria $(b, 0)$ and $\left(u_{0}, w_{0}\right)$, which is called the "waves of invasion"; see Chow and Tam [23].

The paper is organized as follows. In the next section, we first recall a lemma which is a variant of Wazewski's Theorem and then we state the result on the existence of traveling wave solution. Section 3 is devoted to prove the result.

\section{Main Result}

In order to establish the existence of traveling wave solution of the system (6), we assume that the solution has the special form $u(x, t)=u(x+c t), w(x, t)=w(x+c t)$, where the wave speed parameter $c$ is positive. Substituting $u(x, t)=u(s)$, $w(x, t)=w(s), s=x+c t$ into the system (6), the responding system becomes

$$
\begin{gathered}
c u^{\prime}=\alpha u(b-u)-\frac{u w}{1+u^{2}}, \\
c w^{\prime}=w^{\prime \prime}-w+\frac{\beta u w}{1+u^{2}} .
\end{gathered}
$$

Here' denotes the differentiation with respect to the variable $s$. We require that the traveling wave solutions $u$ and $w$ are nonnegative and satisfy the boundary conditions

$$
\begin{gathered}
u(-\infty)=b, \quad u(+\infty)=u_{0} \\
w(-\infty)=0, \quad w(+\infty)=w_{0} .
\end{gathered}
$$

We write the system (6) as a first order system in $R^{3}$

$$
\begin{gathered}
u^{\prime}=\frac{\alpha}{c} u(b-u)-\frac{u w}{c\left(1+u^{2}\right)}, \\
w^{\prime}=z, \quad z^{\prime}=c z+w-\frac{\beta u w}{1+u^{2}} .
\end{gathered}
$$

In this section a variant of Wazewski's Theorem, which is a formalization and extension of the shooting method, is stated. 
This proposition recognizes that the flow defined by the solutions of a differential system gives a topological mapping between regions of phase space. The statement and the proof of Wazewski's Theorem are given in [24].

Consider a system

$$
y^{\prime}=f(y), \quad{ }^{\prime}=\frac{d}{d s}, \quad y \in R^{n}
$$

Here $f: R^{n} \rightarrow R^{n}$ is a continuous function and satisfies the Lipschitz condition. Let $y\left(s, y_{0}\right)$ be the unique solution of $(*)$ satisfying $y\left(0, y_{0}\right)=y_{0}$. For convenience, we set $y\left(s, y_{0}\right)=$ $y_{0} \cdot s$; let $Y \cdot S$ be the set of points $y_{0} \cdot s$, where $y_{0} \in Y$ and $s \in S$.

Given $W \subseteq R^{n}$, the notation $\mathrm{cl}(W)$ is used for the closure of $W$. Define

$$
W^{-}=\left\{y_{0} \in W \mid \forall s>0, y_{0} \cdot[0, s) \nsubseteq W\right\}
$$

$W^{-}$is called the immediate exit set of $W$. Given $\Sigma \subset W$, let

$$
\Sigma^{0}=\left\{y_{0} \in \Sigma \mid \exists s_{0}=s_{0}\left(y_{0}\right) \text { such that } y_{0} \cdot s_{0} \notin W\right\} .
$$

For $y_{0} \in \Sigma^{0}$, define

$$
T\left(y_{0}\right)=\sup \left\{s \mid y_{0} \cdot[0, s] \subseteq W\right\}
$$

$T\left(y_{0}\right)$ is called an exit time. Note that $y_{0} \cdot T\left(y_{0}\right) \in W^{-}$and $T\left(y_{0}\right)=0$ if and only if $y_{0} \in W^{-}$.

\section{Lemma 1. Suppose that}

(i) if $y_{0} \in \Sigma$ and $y_{0} \cdot[0, s] \subseteq \operatorname{cl}(\mathrm{W})$, then $y_{0} \cdot[0, s] \subseteq W$;

(ii) if $y_{0} \in \Sigma, y_{0} \cdot s \in W, y_{0} \cdot s \notin W^{-}$, then there is an open set $V_{s}$ about $y_{0} \cdot s$ disjoint from $W^{-}$;

(iii) $\Sigma=\Sigma^{0}, \Sigma$ is a compact set and intersects a trajectory of $y^{\prime}=f(y)$ only once.

Then the mapping $F\left(y_{0}\right)=y_{0} \cdot T\left(y_{0}\right)$ is a homeomorphism from $\Sigma$ to its image on $W^{-}$. The proof is given in [22]. A set $W \subseteq R^{n}$ satisfying the conditions ( $i$ ) and (ii) is called a Wazewski set.

Theorem 2. (i) If $\beta>\left(1+b^{2}\right) / b$, and $0<c<$ $2 \sqrt{\left(\beta b-1-b^{2}\right) /\left(1+b^{2}\right)}$, then there are no nonnegative solutions of the system (10) satisfying the boundary conditions (9).

(ii) If $\beta>\left(1+b^{2}\right) / b, c>\sqrt{2 \beta-4}, \alpha\left(1+b^{2}\right)<c^{2}$, and $b<2 \sqrt{1+u_{0}^{2}}-u_{0}$, then there exists nonnegative solution of the system (10) satisfying the boundary conditions (9), which correspond to traveling wave solution of the system (6).

\section{Proofs of the Result}

The eigenvalues of the linearization of the system $(10)$ at $(b, 0$, $0)$ are

$$
\begin{gathered}
\lambda_{1}=-\frac{\alpha b}{c}, \\
\lambda_{2}=\frac{c-\sqrt{c^{2}-4\left(\beta b-1-b^{2}\right) /\left(1+b^{2}\right)}}{2}, \\
\lambda_{3}=\frac{c+\sqrt{c^{2}-4\left(\beta b-1-b^{2}\right) /\left(1+b^{2}\right)}}{2} .
\end{gathered}
$$

If $0<c<2 \sqrt{\left(\beta b-1-b^{2}\right) /\left(1+b^{2}\right)}$, then $\lambda_{2}$ and $\lambda_{3}$ are a pair of complex conjugate eigenvalues with positive real part. By Theorems 6.1 and 6.2 in [16], there is a two-dimensional unstable manifold base at $(b, 0,0)$; the critical point is a spiral point on this unstable manifold, so the trajectory approaching $(b, 0,0)$ as $s \rightarrow-\infty$ must have $w(s)<0$ for some $s$. It violates the requirement that the solution of the system (10) must be nonnegative. It proves the first part of Theorem 2 .

We only need to discuss the case $c \geq$ $2 \sqrt{\left(\beta b-1-b^{2}\right) /\left(1+b^{2}\right)}$. In fact we require the stronger condition $c>\sqrt{2 \beta-4}$ for mathematical simplicity. With the requirement there are three distinct real eigenvalues $\lambda_{1}<0<\lambda_{2}<\lambda_{3}$. Let the eigenvectors $\mathbf{e}_{1}, \mathbf{e}_{2}, \mathbf{e}_{3}$ associated with $\lambda_{1}, \lambda_{2}, \lambda_{3}$, respectively, be

$$
\begin{gathered}
\mathbf{e}_{1}=(1,0,0), \quad \mathbf{e}_{2}=\left(1, p\left(\lambda_{2}\right), \lambda_{2} p\left(\lambda_{2}\right)\right), \\
\mathbf{e}_{3}=\left(1, p\left(\lambda_{3}\right), \lambda_{3} p\left(\lambda_{3}\right)\right) .
\end{gathered}
$$

Here $p\left(\lambda_{i}\right)=-(1 / b)\left(c \lambda_{i}+c b^{2} \lambda_{i}+\alpha b+\alpha b^{3}\right)<0, i=2,3$.

Applying Theorems 6.1 and 6.2 of [16], there exists a onedimension strongly unstable manifold $\Omega_{1}$ tangent to $\mathbf{e}_{3}$ at $(b$, $0,0)$. A parametric representation for the strongly unstable manifold $\Omega_{1}$ in a small neighborhood of $(b, 0,0)$ is

$$
f_{1}(m)=(b, 0,0)+m \cdot \mathbf{e}_{3}+O(|m|) .
$$

There exists a two-dimension unstable manifold $\Omega_{2}$ tangent to the span of $\mathbf{e}_{2}$ and $\mathbf{e}_{3}$ at $(b, 0,0)$. A parametric representation for the two-dimensional unstable manifold $\Omega_{2}$ in a small neighborhood of $(b, 0,0)$ is

$$
f_{2}(m, n)=(b, 0,0)+m \cdot \mathbf{e}_{3}+n \cdot \mathbf{e}_{2}+O(|m|+|n|) .
$$

The idea of constructing the Wazewski set $W$ is similar to that in Dunbar [22]: it will be the complement of three blocks in $R^{3}$, two of which are chosen so that $z^{\prime}$ has the same sign as $z$ so solutions entering these blocks would not have $z \rightarrow 0$ as $s \rightarrow \infty$. Thus we define the Wazewski set $W$ as follows:

$$
W=R^{3} \backslash(P \cup Q) \text {. }
$$

Here

$$
\begin{aligned}
& P=\left\{(u, w, z) \mid u<u_{0}, w>w_{0}, z>0\right\} \\
& Q=\left\{(u, w, z) \mid u>u_{0}, w<w_{0}, z<0\right\} .
\end{aligned}
$$


Note that $W$ is a closed set. Let

$$
\begin{aligned}
J= & \left\{(u, v, w, z) \mid u>u_{1}, 0 \leq w \leq w_{0}, z=0\right\} \\
& \cup\left\{(u, v, w, z) \mid u_{0} \leq u<u_{1}, w \leq 0, z=0\right\} \\
& \cup\left\{(u, v, w, z) \mid u=u_{1}, w_{1} \leq w \leq 0, z=0\right\} .
\end{aligned}
$$

By checking the vector field on $\partial W$, we obtain

$$
W^{-}=\partial W \backslash\left(J \cup\left\{\left(u_{0}, w_{0}, 0\right)\right\}\right) .
$$

Details of proof that $W^{-}$is the set described above are tedious. We only examine the part $\partial Q$ of $\partial W$ as an example, which shows why the set $J$ must be excluded from $\partial W$ to obtain $W^{-}$. The other proofs are similar. The boundary of $\partial Q$ is $u=u_{0}$, $w=w_{0}$, or $z=0$.

(1) $u=u_{0}, w=w_{0}$, and $z<0$.

Since $w^{\prime}=z<0, w<w_{0}, u^{\prime}=[(\alpha / c) u(b-u)-$ $\left.u w / c\left(1+u^{2}\right)\right]_{\left(u_{0}, w_{0}\right)}=0, u^{\prime \prime}=-\left(u_{0} z / c\left(1+u_{0}^{2}\right)\right)>0$, and $u>u_{0}$, thus the trajectory enters $Q$.

(2) $u=u_{0}, w<w_{0}$, and $z=0$.

Since $u^{\prime}=\left[(\alpha / c) u(b-u)-u w / c\left(1+u^{2}\right)\right]_{u=u_{0}}>0$, $z^{\prime}=\left[c z+w\left(1-\beta u /\left(1+u^{2}\right)\right)\right]_{u=u_{0}}=0, z^{\prime \prime}=\beta u^{\prime} w\left(u_{0}^{2}-\right.$ $1) /\left(1+u_{0}^{2}\right)^{2}$, and $u_{0}<1$, thus $u>u_{0}$ and we obtain the following.

(i) $0<w<w_{0}$; then $z^{\prime \prime}<0, z<0$, and the trajectory enters $Q$.

(ii) $w<0$; then $z^{\prime \prime}>0, z>0$, and the trajectory does not enter $Q$.

(iii) $w=0, z=0$. Consider the system

$$
w^{\prime}=z, \quad z^{\prime}=c z+w-\frac{\beta u w}{1+u^{2}} .
$$

We come to the conclusion that the $u$-axis is an invariant manifold and the trajectory does not enter $Q$.

(3) $u>u_{0}, w=w_{0}$, and $z=0$.

Since $u_{1}=\left(\beta+\sqrt{\beta^{2}-4}\right) / 2>b$ and $z^{\prime}=w_{0}(1-$ $\left.\beta u /\left(1+u^{2}\right)\right)$, we obtain the following.

(i) $u_{0}<u<u_{1}$; then $z^{\prime}<0, z<0, w^{\prime \prime}=z^{\prime}<0$, $w<w_{0}$, and the trajectory enters $Q$.

(ii) $u>u_{1}$; then $z^{\prime}>0, z>0, w^{\prime \prime}=z^{\prime}>0, w>w_{0}$, and the trajectory does not enter $Q$.

(iii) $u=u_{1}$; then $u^{\prime}=(\alpha / c) u_{1}\left(b-u_{1}\right)-u_{1} w_{0} / c(1+$ $\left.u_{1}^{2}\right)<0, z^{\prime}=0$, and $z^{\prime \prime}=\left[w-\beta u w /\left(1+u^{2}\right)\right]^{\prime}=$ $\beta u^{\prime} w_{0}\left(u_{1}^{2}-1\right) /\left(1+u_{1}^{2}\right)^{2}<0$.

That is, $z<0, w<w_{0}$, and the trajectory enters $Q$.

(4) $u=u_{0}, w<w_{0}$, and $z<0$.

From the proof of (2), we come to the conclusion that $u^{\prime}>0, u>u_{0}$, and the trajectory enters $Q$.

(5) $u>u_{0}, w=w_{0}$, and $z<0$.
Since $w^{\prime}=z<0$ and $w<w_{0}$, the trajectory enters $Q$.

(6) $u_{0}<u<u_{1}, w<w_{0}$, and $z=0$.

Since $z^{\prime}=w\left(1-\beta u /\left(1+u^{2}\right)\right)$, we obtain the following.

(i) $0<w<w_{0}$; then $z^{\prime}<0$ and $z<0$, which implies that the trajectory enters $Q$.

(ii) $w=0$; similar to the proof of (2iii), the trajectory does not enter $Q$.

(iii) $w<0$; then $z^{\prime}>0$ and $z>0$; that is, the trajectory does not enter $Q$.

(7) $u=u_{1}, w<w_{0}$, and $z=0$.

Since $u^{\prime}=(\alpha / c) u_{1}\left(b-u_{1}\right)-u_{1} w / c\left(1+u_{1}^{2}\right), z^{\prime}=[c z+$ $\left.w\left(1-\beta u /\left(1+u^{2}\right)\right)\right]_{u=u_{1}}=0$, and $z^{\prime \prime}=\beta u^{\prime} w\left(u_{1}^{2}-\right.$ $1) /\left(1+u_{1}^{2}\right)^{2}$, we obtain the following.

(i) $0<w<w_{0}$; then $u^{\prime}<0, z^{\prime \prime}<0, z<0$, and the trajectory enters $Q$.

(ii) $w=0$; similar to the proof of (2iii), the trajectory does not enter $Q$.

(iii) $w_{1}<w<0$; then $u^{\prime}<0$ and $z^{\prime \prime}>0$; that is, $z>0$, and the trajectory does not enter $Q$.

(iv) $w=w_{1}$. It is a singular point $\left(u_{1}, w_{1}, 0\right)$ and is not in the immediate exit set.

(v) $w<w_{1}$; then $u^{\prime}>0, z^{\prime \prime}<0$, and $z<0$, which implies that the trajectory enters $Q$.

(8) $u>u_{1}, w<w_{0}$, and $z=0$.

Since $z^{\prime}=w\left(1-\beta u /\left(1+u^{2}\right)\right)$, then we obtain the following.

(i) $0<w<w_{0}$; then $z^{\prime}>0, z>0$, and the trajectory does not enter $Q$.

(ii) $w=0$; the trajectory does not enter $Q$.

(iii) $w<0$; then $z^{\prime}<0, z<0$, and the trajectory enters $Q$.

In order to use Lemma 1, we construct the set $\Sigma$ on a sphere surrounding $(b, 0,0)$ in the two-dimensional unstable manifold $\Omega_{2}$ by Lemma 3 to Lemma 7 . The specification of the arc requires the identification of the endpoints on the circle. One endpoint is the intersection of the circle with the strongly unstable manifold $\Omega_{1}$ and the other is the intersection of the circle with the plane defined by $z=0$. Lemmas 3-6 are simple comparison arguments showing that the first endpoint on the strongly unstable manifold is carried by the flow into $P$ and the other is carried into $Q$. We use the notation $\Lambda_{1}=\{(u, w, z) \mid u \leq b, w \geq 0, z \geq 0\}$.

Lemma 3. Let $c>\sqrt{2 \beta-4}$. A solution of the system (10) having a point, corresponding to $s=0$ without loss of generality, such that $u(0)<b, w(0)>0$, and $z(0)>(c / 2) w(0)$, will have $w(s)>0$ and $z(s)>(c / 2) w(s)$ for all $s>0$. In particular, it is true for trajectories on the branch of strongly unstable manifold $\Omega_{1}$ in the octant $\Lambda_{1}$. 
Proof. Suppose, to the contrary, that there exists an $s>0$ such that $u(s)<b$, but $z(s) \leq(c / 2) w(s)$. Let $s_{1}=\inf \{s \mid$ $z(s) \leq(c / 2) w(s), u(s)<b\}$. Since $w(0)>0$ and for $s \in\left[0, s_{1}\right)$, $w^{\prime}(s)=z(s)>(c / 2) w(s)$, we have $w\left(s_{1}\right)>0$ and $z^{\prime}\left(s_{1}\right)-$ $(c / 2) w^{\prime}\left(s_{1}\right) \leq 0$. Using that $z\left(s_{1}\right)=(c / 2) w\left(s_{1}\right)$, we obtain $\left(c^{2} / 4\right)+1-\beta u\left(s_{1}\right) /\left(1+u^{2}\left(s_{1}\right)\right) \leq 0$. Since $u\left(s_{1}\right) /\left(1+u^{2}\left(s_{1}\right)\right) \leq$ $1 / 2$, it follows that $c^{2} / 4+1-\beta / 2 \leq 0$; that is, $c^{2} \leq 2 \beta-4$; it is a contradiction with $c^{2}>2 \beta-4$. It completes the proof.

Lemma 4. A trajectory on the portion of strongly unstable manifold $\Omega_{1}$ in the octant $\Lambda_{1}$ must satisfy

$$
w(s) \geq-c^{2}(u(s)-b)
$$

for all s.

Proof. The solution approaches $(b, 0,0)$ tangent to $\mathbf{e}_{3}$ and the eigenvector $\mathbf{e}_{3}$ at $(b, 0,0)$ has $w=p\left(\lambda_{3}\right)(u-b)$ such that $w(0)>-c^{2}(u(0)-b)$. Suppose to the contrary that there exists an $s>0$ such that $w(s)<-c^{2}(u(s)-b)$. Let $s_{1}=\inf \{s \mid w(s)<$ $\left.-c^{2}(u(s)-b)\right\}$; then $w^{\prime}\left(s_{1}\right)<-c^{2} u^{\prime}\left(s_{1}\right)$. Since $u^{\prime}=(\alpha / c) u(b-$ $u)-u w / c\left(1+u^{2}\right), w^{\prime}=z$, it follows that $z\left(s_{1}\right) \leq(c / 2) w\left(s_{1}\right)$; it is a contradiction. It completes the proof.

Lemma 5. Let $d>\left(c+\sqrt{c^{2}+4}\right) / 2$ be a fixed number. $A$ solution of the system (10) having a point, corresponding to $s=0$ without loss of generality, such that $0<u(0)<b$ and $z(0)<d w(0)$, will have $z(s)<d w(s)$ for all $s>0$ such that $w(s)>0$. In particular, this is true for trajectories on the branch of strongly unstable manifold $\Omega_{1}$ in the octant $\Lambda_{1}$.

Proof. Suppose, to the contrary, that there exists an $s>0$ such that $w(s)>0$, but $z(s) \geq d w(s)$. Let $s_{1}=\inf \{s>0 \mid$ $z(s) \geq d w(s)\}$; then $z\left(s_{1}\right)=d w\left(s_{1}\right)$ and $z(s)<d w(s)$ for $0 \leq s<s_{1} z^{\prime}\left(s_{1}\right)-d w^{\prime}\left(s_{1}\right) \geq 0$. Substituting $z^{\prime}$ and $w^{\prime}$, we obtain $c z\left(s_{1}\right)-d z\left(s_{1}\right)+w\left(s_{1}\right)-\beta u\left(s_{1}\right) w\left(s_{1}\right) /\left(1+u^{2}\left(s_{1}\right)\right) \geq 0$; that is, $\left[-d^{2}+c d+1-\beta u\left(s_{1}\right) /\left(1+u^{2}\left(s_{1}\right)\right)\right] w\left(s_{1}\right) \geq 0$. However, the choice of $d$ implies that $-d^{2}+c d+1<0$; thus $\left[-d^{2}+c d+1-\beta u\left(s_{1}\right) /\left(1+u^{2}\left(s_{1}\right)\right)\right] w\left(s_{1}\right)<0$. This contradiction shows that $z(s)<d w(s)$ for $s$ such that $w(s)>0$.

Lemma 6. Suppose that a solution of the system (10) has a point such that

$$
\begin{gathered}
u(0)<b, \quad 0<w(0)<-\frac{(d c+\alpha b)\left(1+b^{2}\right)}{u_{0}}(u(0)-b), \\
z(0)<d w(0) .
\end{gathered}
$$

Then for all $s>0$, as long as $u(s)>u_{0}, w(s)>0$, the trajectory must satisfy that

$$
w(s)<-\frac{(d c+\alpha b)\left(1+b^{2}\right)}{u_{0}}(u(s)-b) .
$$

In particular, it is true for trajectories on the branch of strongly unstable manifold $\Omega_{1}$ in the octant $\Lambda_{1}$.
Proof. We first show that $u(s)<b$ for all $s>0$ such that $w(s)>0$. If it is not true, then there is a first $s_{1}$ such that $u\left(s_{1}\right)=b, u^{\prime}\left(s_{1}\right) \geq 0$, and $w\left(s_{1}\right)>0$. However, we have

$$
u^{\prime}\left(s_{1}\right)=\frac{u}{c}\left[\alpha(b-u)-\frac{w}{1+u^{2}}\right]_{s=s_{1}}<0 .
$$

It is a contradiction; then $u(s)<b$ for all $s$ such that $w(s)>0$.

Let $A=(d c+\alpha b)\left(1+b^{2}\right) / u_{0}$; suppose that there exists a first time $s_{2}>0$ such that $u\left(s_{2}\right)>u_{0}, w\left(s_{2}\right)>0$, and $w\left(s_{2}\right)=$ $-A\left(u\left(s_{2}\right)-b\right)$; then $w^{\prime}\left(s_{2}\right) \geq-A u^{\prime}\left(s_{2}\right)$. By Lemma 5 , we obtain

$$
d w\left(s_{2}\right) \geq z\left(s_{2}\right) \geq-\frac{A u}{c}\left[\alpha(b-u)-\frac{w}{1+u^{2}}\right]_{s=s_{2}} .
$$

From $w\left(s_{2}\right)=-A\left(u\left(s_{2}\right)-b\right)$ and $u_{0}<u\left(s_{2}\right)<b$, we have

$$
d \geq-\frac{1}{c}\left[\alpha u-\frac{A u}{1+u^{2}}\right]_{s=s_{2}}>\frac{1}{c}\left[-\alpha b+\frac{A u_{0}}{1+b^{2}}\right]=d .
$$

It is a contradiction, which completes the proof.

Combining the results of these lemmas, we follow the trajectory of a solution of the system (10) on the strongly unstable manifold $\Omega_{1}$. Define

$$
\begin{aligned}
& \Re=\left\{(u, w, z) \mid u_{0}<u<b,\right. \\
& -c^{2}(u(s)-b) \leq w \\
& \leq-\frac{(d c+\alpha b)\left(1+b^{2}\right)(u(s)-b)}{u_{0}}, \\
& \left.\frac{c}{2} w<z<d w\right\} .
\end{aligned}
$$

Lemmas 3-6 show that the trajectory of a solution of the system (10) on the strongly unstable manifold $\Omega_{1}$ is contained in $\Re$. Recall the assumption that $\alpha<c^{2} /\left(1+b^{2}\right)$; then

$$
w \geq c^{2}(b-u(s))>\alpha\left(1+u^{2}\right)(b-u(s)),
$$

which implies that $u^{\prime}<0$ in the region $\mathfrak{R}$. Thus, for a solution of the system (10) on the strongly unstable manifold $\Omega_{1}, u(s)$ decreases until $u\left(s_{1}\right)=u_{0}$ for some finite $s_{1}$; the trajectory of this solution hits $\partial P$ on the face $u=u_{0}, w>w_{0}$, and $z>$ 0 . The vector field on this face shows that a solution of the system (10) on $\Omega_{1}$ enters the region $P$ at some finite time.

Lemma 7. In a sufficiently small neighborhood of $(b, 0,0)$, the two-dimensional unstable manifold $\Omega_{2}$ intersects the plane defined by $z=0$ in a $C^{1}$ curve $\Gamma$, given by $w=J(u)$ and $z=0$.

Proof. The proof, which is similar to that of Lemma 5 in [13], is therefore omitted.

We are interested in the portion of the curve $\Gamma$ in the region $u<b$. The function $J(u)$ can be approximated to the first order by

$$
w=J(u)=\frac{p\left(\lambda_{2}\right) p\left(\lambda_{3}\right)\left(\lambda_{3}-\lambda_{2}\right)}{\lambda_{3} p\left(\lambda_{3}\right)-\lambda_{2} p\left(\lambda_{2}\right)}(u-b) .
$$


Thus the $w$-coordinate of points along the curve $\Gamma$ will satisfy $w>0$. From the direction of the vector field on the plane defined by $u_{0}<u<b, w>0$, and $z=0$, any trajectory passing through a point of $\Gamma$ near $(b, 0,0)$ will immediately enter the region $Q$.

Now, we place a sufficiently small circle surrounding (b, $0,0)$ on the two-dimensional unstable manifold $\Omega_{2}$ such that the circle is contained in the neighborhood of $(b, 0,0)$ given in Lemma 7 and the conditions of Lemmas 3-6 are satisfied. The circle intersects the curve $\Gamma$. Define $\Sigma$ to be the arc of this circle contained in the octant $\Lambda_{1}$, whose endpoints are the intersections of the circle with $\Omega_{1}$ and the curve $\Gamma$.

We now prove part (ii) of Theorem 2 , which requires two steps. Firstly, we use Lemma 1 to produce a trajectory which remains in the region $W$. Secondly, we construct a Lyapunov function to prove the trajectory approaches $\left(u_{0}, w_{0}, 0\right)$.

Lemma 8. There exists a point $y^{*} \in \Sigma$ such that the solution $y\left(s, y^{*}\right)$ of the system (10) remains in the region $W$ for all $s$.

Proof. The proof, which is similar to the proof of Lemma 3.7 in [15], is therefore omitted.

Lemma 9. The solution $y\left(s, y^{*}\right)$ must be in the bounded region

$$
\Psi=\left\{(u, w, z) \mid 0<u<b, 0<w<k(u),-\frac{1}{c} w<z<d w\right\}
$$

for all s, where

$$
k(u)=\left\{\begin{aligned}
-\frac{(d c+\alpha b)\left(1+b^{2}\right)(u-b)}{u_{0}}, & u_{0}<u<b, \\
-\frac{(d c+\alpha b)\left(1+b^{2}\right)\left(u_{0}-b\right)}{u_{0}}, & 0<u \leq u_{0} .
\end{aligned}\right.
$$

Proof. Since the plane defined by $u=0$ is an invariant manifold, the first coordinate of $y_{1}$ is strictly positive, and thus $u_{1}>0$ for all $s$. Suppose $y\left(s, y^{*}\right)$ enters the region $N_{1}=\{(u, w, z) \mid w \leq 0\} ;$ let $s_{1}=\inf \left\{s \mid y\left(s, y^{*}\right) \in N_{1}\right\}$; then we have $w_{1}\left(s_{1}\right)=0, w_{1}^{\prime}\left(s_{1}\right) \leq 0$, and $z_{1}\left(s_{1}\right) \leq 0$. We know that the $u$-axis is an invariant manifold, $z_{1}\left(s_{1}\right)<0$. Since $y\left(s, y^{*}\right)$ does not enter $Q$ then $u_{1}\left(s_{1}\right)<u_{0}$. From the system (10), we obtain $u_{1}^{\prime}\left(s_{1}\right)>0$, which must enter the region

$$
N_{2}=\left\{(u, w, z) \mid u_{1}\left(s_{1}\right)<u<u_{0}, w<0, z<0\right\} .
$$

In the region $N_{2}, z_{1}(s)$ and $w_{1}(s)$ are decreasing, and thus $u_{1}^{\prime}(s)$ is bounded below by the positive

$$
\frac{\alpha}{c} \min \left\{u_{1}\left(s_{1}\right)\left(b-u_{1}\left(s_{1}\right)\right), u_{0}\left(b-u_{0}\right)\right\} .
$$

Then $u_{1}(s)$ increases to $u_{0}$ in a finite time and $y\left(s, y^{*}\right)$ enters Q. It is a contradiction, so $w_{1}(s)>0$ for all $s$.

From Lemma 6, we obtain that $w_{1}<-(d c+\alpha b)(1+$ $\left.b^{2}\right)\left(u_{1}-b\right) / u_{0}$ for $u_{0}<u_{1} \leq b$. Since $w_{1}(s)>0$, we have $u_{1}\left(s_{1}\right)<b$ for all $s$. Suppose that there is an $s$ such that $w_{1}(s) \geq$ $-A_{0}\left(u_{0}-b\right)$ for $0<u_{1} \leq u_{0}$, where $A_{0}=(d c+\alpha b)\left(1+b^{2}\right) / u_{0}$.
Let $s_{2}=\inf \left\{s \mid w_{1}(s) \geq-A_{0}\left(u_{0}-b\right)\right\}$, so $u_{1}\left(s_{2}\right) \leq u_{0}, w_{1}\left(s_{2}\right)>$ $w_{0}$, and $z_{1}\left(s_{2}\right)=w_{1}^{\prime}\left(s_{2}\right) \geq 0$. Then either $y\left(s_{2}, y^{*}\right) \in P$ or $y\left(s_{2}, y^{*}\right)$ immediately enters $P$, which is a contradiction.

Suppose that there exists an $s_{3}$ such that $z_{1}\left(s_{3}\right)<$ $-(1 / c) w_{1}\left(s_{3}\right)<0$; then $z_{1}(s)<-(1 / c) w_{1}(s)$ for all $s>s_{3}$. If it is not true, there exists an $s_{4}>s_{3}$ such that $z_{1}\left(s_{4}\right)=$ $-(1 / c) w_{1}\left(s_{4}\right)$, and thus $z_{1}^{\prime}\left(s_{4}\right)+(1 / c) w_{1}^{\prime}\left(s_{4}\right) \geq 0$. From the system (10), we have

$$
\frac{1}{c^{2}}+\frac{\beta u}{1+u^{2}} \leq 0,
$$

which is impossible. So if $z_{1}\left(s_{3}\right)<-(1 / c) w_{1}\left(s_{3}\right)<0$, then $z_{1}(s)<-(1 / c) w_{1}(s)$ continues to hold for $s>s_{3}$. Thus, $z_{1}^{\prime}=$ $c z_{1}+w_{1}-\beta u_{1} w_{1} /\left(1+u_{1}^{2}\right)<-\beta u_{1} w_{1} /\left(1+u_{1}^{2}\right)<0$ and $z_{1}(s)<$ $z_{1}\left(s_{3}\right)$ for all $s>s_{3}$ and $w_{1}^{\prime}(s)=z_{1}(s)$ is strictly negative and bounded away from zero by $z_{1}\left(s_{3}\right)$. Then $w_{1}(s)<0$ for some finite $s$; it is a contradiction. Notice that a trajectory starting on $\Sigma$ tangent to $\mathbf{e}_{2}$ or $\mathbf{e}_{3}$ has $z=\lambda_{2} w$ or $z=\lambda_{3} w$. Since $\lambda_{2}$ and $\lambda_{3}<d$, we have $z_{1}(s)<d w_{1}(s)$ for all $s$, which completes the proof of this lemma.

Lemma 10. The trajectory $y\left(s, y^{*}\right) \rightarrow\left(u_{0}, w_{0}, 0\right)$ as $s \rightarrow$ $+\infty$.

Proof. In order to show the trajectory will approach the point $\left(u_{0}, w_{0}, 0\right)$, we construct a Lyapunov function as follows:

$$
\begin{aligned}
V(u, w, z)= & c\left[\left(1+u_{0}^{2}\right) u-u_{0} \ln u-\frac{u_{0}}{2} u^{2}\right] \\
& +u_{0}\left[c\left(w-w_{0}\right)-z\right]+w_{0} u_{0}\left(\frac{z}{w}-c \ln \frac{w}{w_{0}}\right) .
\end{aligned}
$$

We obtain that $V(u, w, z)$ is continuous and bounded below on $\Psi$,

$$
\begin{aligned}
\frac{d V}{d s}= & \frac{\partial V}{\partial u} u_{t}+\frac{\partial V}{\partial w} w_{t}+\frac{\partial V}{\partial z} z_{t} \\
= & \alpha\left(u-u_{0}\right)\left(1-u_{0} u\right)(b-u)+\frac{\left(u-u_{0}\right)\left(u_{0} u-1\right)}{1+u^{2}} w \\
& +\left(w_{0}-w\right) \frac{\left(u-u_{0}\right)\left(u_{0} u-1\right)}{1+u^{2}}-\frac{w_{0} u_{0}}{w^{2}} z^{2} \\
= & \left(u-u_{0}\right)\left(u_{0} u-1\right)\left[\frac{w_{0}}{1+u^{2}}-\alpha(b-u)\right]-\frac{w_{0} u_{0}}{w^{2}} z^{2} \\
= & \left(u-u_{0}\right)\left(u_{0} u-1\right) f(u)-\frac{w_{0} u_{0}}{w^{2}} z^{2}
\end{aligned}
$$

Here $f(u)=w_{0} /\left(1+u^{2}\right)-\alpha(b-u)$. Recall the assumption that $b<2 \sqrt{1+u_{0}^{2}}-u_{0}$; then

$$
\left(u-u_{0}\right)\left(u_{0} u-1\right) f(u) \leq 0 .
$$

Therefore, $d V / d s$ is always nonpositive in $\Psi$. Moreover, $d V / d s=0$ if and only if $z=0, u=u_{0}$, and the largest invariant subset of this segment is the single point 
$\left(u_{0}, w_{0}, 0\right)$. By LaSalle's invariance principle, it follows that $y(s) \rightarrow\left(u_{0}, w_{0}, 0\right)$ as $s \rightarrow+\infty$, which completes the proof of Theorem 2.

\section{Conflict of Interests}

The authors declare that there is no conflict of interests regarding the publication of this paper.

\section{Acknowledgments}

The authors are grateful to Zhengyi Lu for discussing the research and his useful remarks. The research was partially supported by the Program of Chongqing Innovation Team Project in University under Grant no. KJTD201308 and the Program of Chongqing Normal University under Grant no. 13XLB015. This work is supported by Grant from the Scientific Research Project of the University of South China (no. 2011XQD36) and supported by the construct program in USC.

\section{References}

[1] J. F. Andrews, "A mathematical model for the continuous culture of microorganisms utilizing inhibitory substrates," Biotechnology and Bioengineering, vol. 10, pp. 707-723, 1968.

[2] W. Sugie and J. A. Howell, "Kinetics of phenol by washed cell," Biotechnology and Bioengineering, vol. 23, pp. 2039-2049, 1980.

[3] A. A. Berryman, "The origins and evolution of predator-prey theory," Ecology, vol. 73, pp. 1530-1535, 1992.

[4] Y.-H. Fan and W.-T. Li, "Global asymptotic stability of a ratiodependent predator-prey system with diffusion," Journal of Computational and Applied Mathematics, vol. 188, no. 2, pp. 205-227, 2006.

[5] S. A. Gourley and N. F. Britton, "A predator-prey reactiondiffusion system with nonlocal effects," Journal of Mathematical Biology, vol. 34, no. 3, pp. 297-333, 1996.

[6] L.-L. Wang and W.-T. Li, "Periodic solutions and permanence for a delayed nonautonomous ratio-dependent predator-prey model with Holling type functional response," Journal of Computational and Applied Mathematics, vol. 162, no. 2, pp. 341-357, 2004.

[7] H. I. Freedman, Deterministic Mathematical Models in Population Ecology, vol. 57, Marcel Dekker, New York, NY, USA, 1980.

[8] R. A. Gardner, "Existence of travelling wave solutions of predator-prey systems via the connection index," SIAM Journal on Applied Mathematics, vol. 44, no. 1, pp. 56-79, 1984.

[9] R. May, Stability and Complexity in Model Ecosystems, Princeton University Press, Princeton, NJ, USA, 1974.

[10] A. Okubo, Diffusion and Ecological Problems: Mathematical Models, vol. 10 of Lectures in Biomathematics, Springer, Berlin, Germany, 1980.

[11] M. R. Owen and M. A. Lewis, "How predation can slow, stop or reverse a prey invasion," Bulletin of Mathematical Biology, vol. 63, pp. 655-684, 2001.

[12] J. Huang, G. Lu, and S. Ruan, "Existence of traveling wave solutions in a diffusive predator-prey model," Journal of Mathematical Biology, vol. 46, no. 2, pp. 132-152, 2003.
[13] S. R. Dunbar, "Travelling wave solutions of diffusive LotkaVolterra equations," Journal of Mathematical Biology, vol. 17, no. 1, pp. 11-32, 1983.

[14] S. R. Dunbar, "Traveling waves in diffusive predator-prey equations: periodic orbits and point-to-periodic heteroclinic orbits," SIAM Journal on Applied Mathematics, vol. 46, no. 6, pp. 1057-1078, 1986.

[15] W.-T. Li and S.-L. Wu, "Traveling waves in a diffusive predatorprey model with Holling type-III functional response," Chaos, Solitons \& Fractals, vol. 37, no. 2, pp. 476-486, 2008.

[16] P. Hartman, Ordinary Differential Equations, John Wiley \& Sons, New York, NY, USA, 1964.

[17] J. P. LaSalle, "Stability theory for ordinary differential equations," Journal of Differential Equations, vol. 4, pp. 57-65, 1968.

[18] R. Seydel, Practical Bifurcation and Stability Analysis: From Equilibrium to Chaos, Springer, Berlin, Germany, 1994.

[19] J. D. Murray, Mathematical Biology, vol. 19, Springer, Berlin, Germany, 2nd edition, 1993.

[20] K. Mischaikow and J. F. Reineck, "Travelling waves in predatorprey systems," SIAM Journal on Mathematical Analysis, vol. 24, no. 5, pp. 1179-1214, 1993.

[21] A. I. Volpert, V. A. Volpert, and V. A. Volpert, Traveling Wave Solutions of Parabolic Systems, vol. 140, American Mathematical Society, Providence, RI, USA, 1994.

[22] S. R. Dunbar, "Traveling wave solutions of diffusive LotkaVolterra equations: a heteroclinic connection in $\mathbf{R}^{4}$," Transactions of the American Mathematical Society, vol. 286, no. 2, pp. 557-594, 1984.

[23] P. L. Chow and W. C. Tam, "Periodic and traveling wave solutions to Volterra-Lotka equations with diffusion," Bulletin of Mathematical Biology, vol. 38, no. 6, pp. 643-658, 1976.

[24] C. Conley, Isolated Invariant Sets and the Morse Index, vol. 38 of CBMS Regional Conference Series in Mathematics, American Mathematical Society, Providence, RI, USA, 1978. 


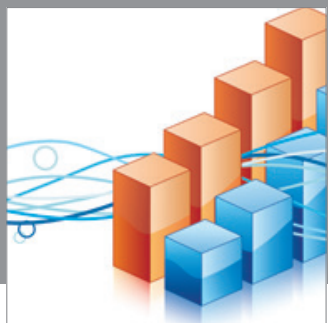

Advances in

Operations Research

mansans

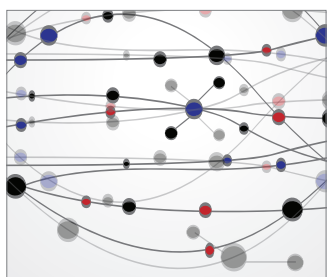

The Scientific World Journal
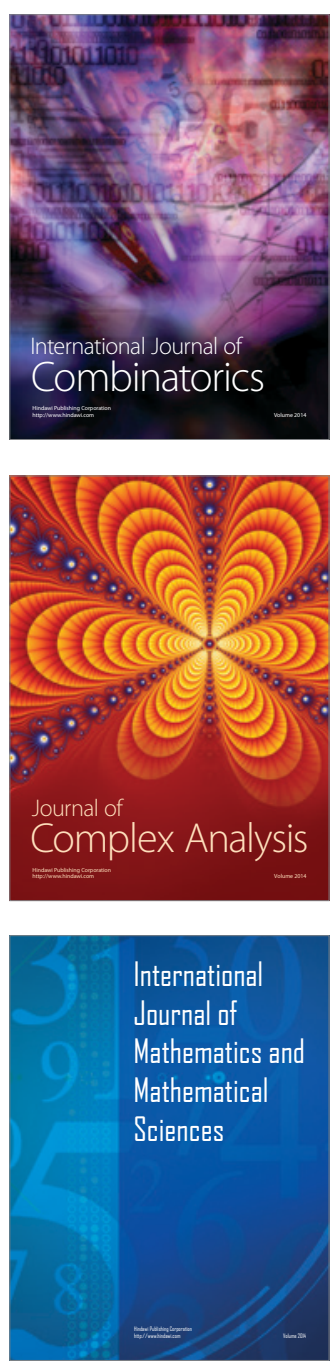
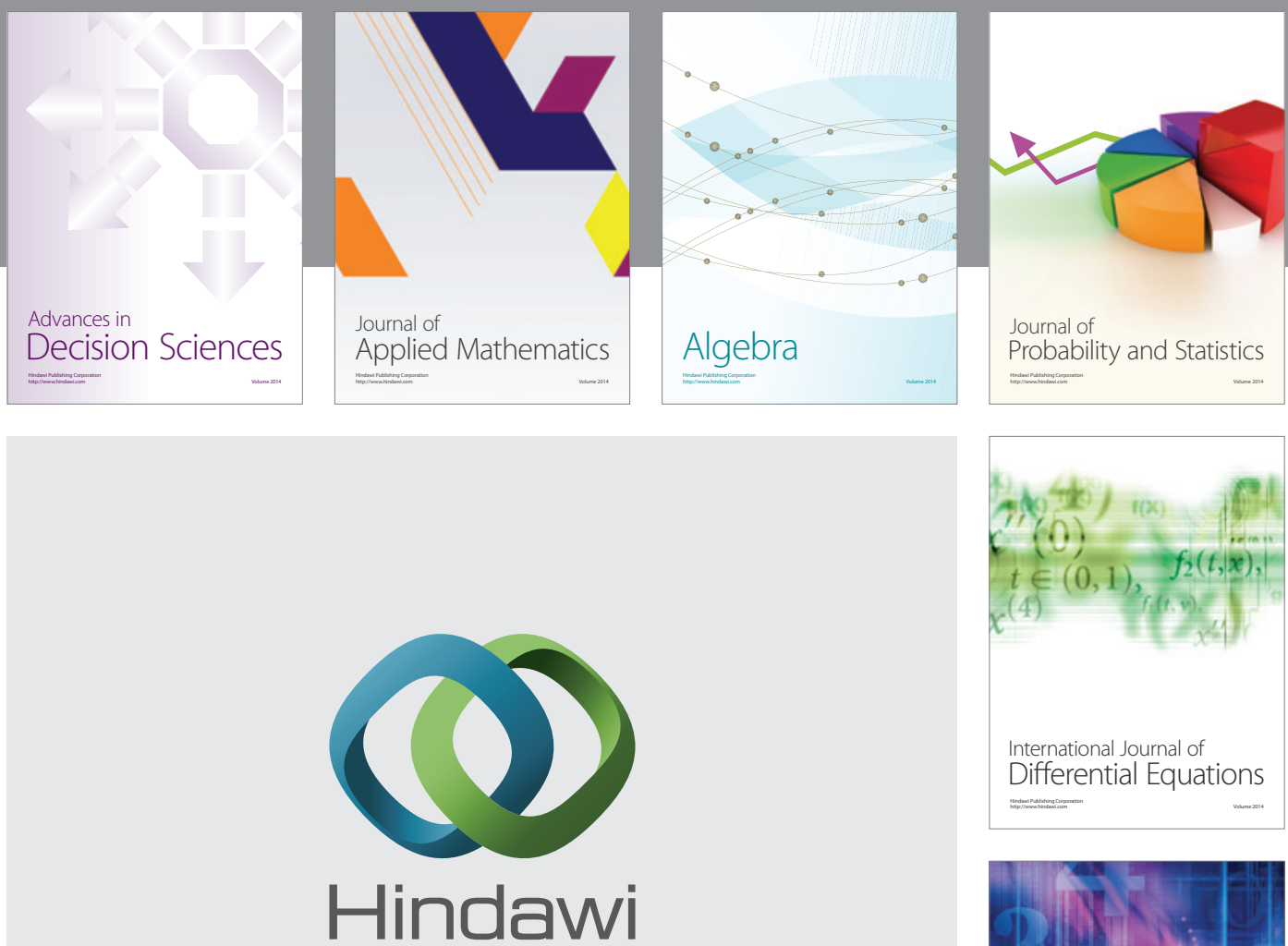

Submit your manuscripts at http://www.hindawi.com
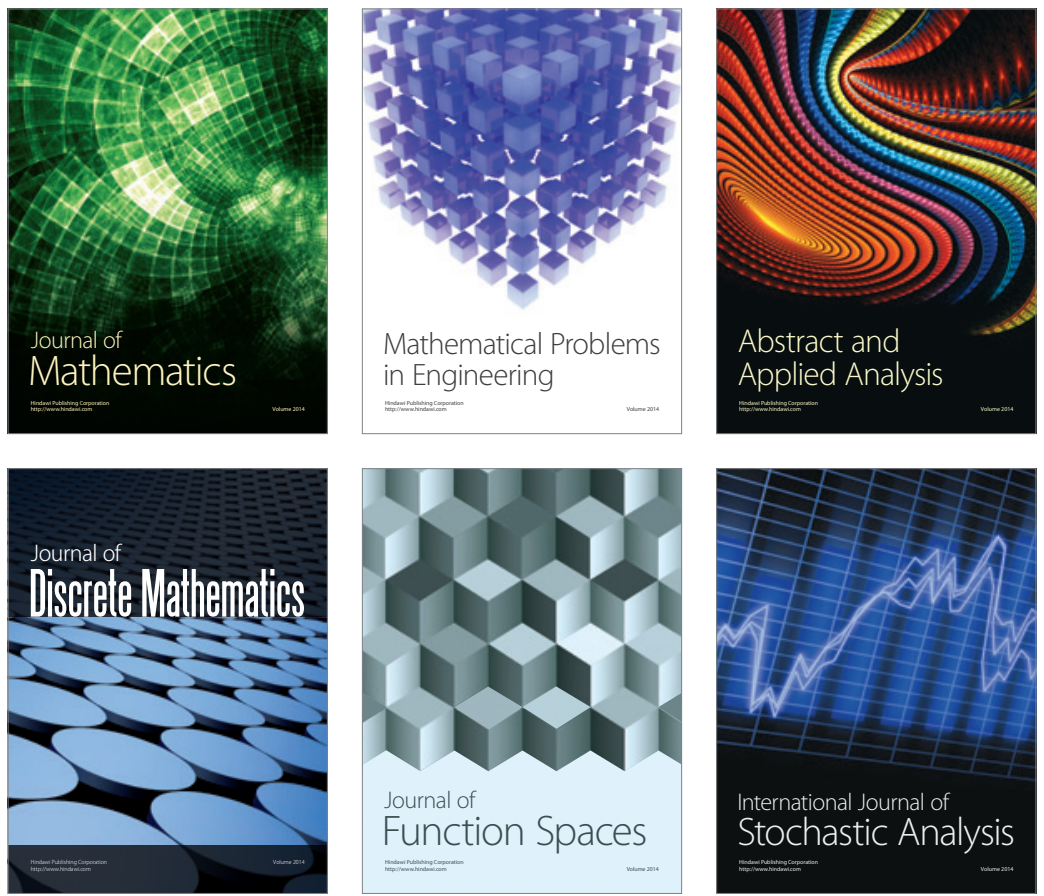

Journal of

Function Spaces

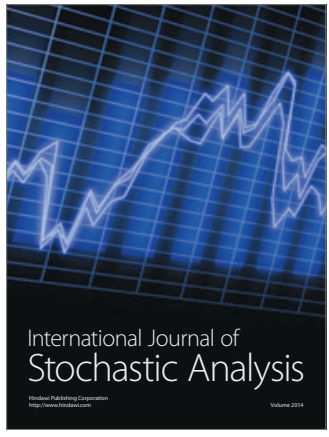

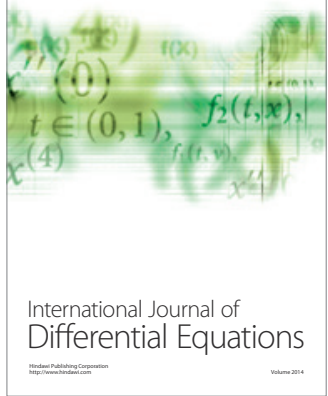
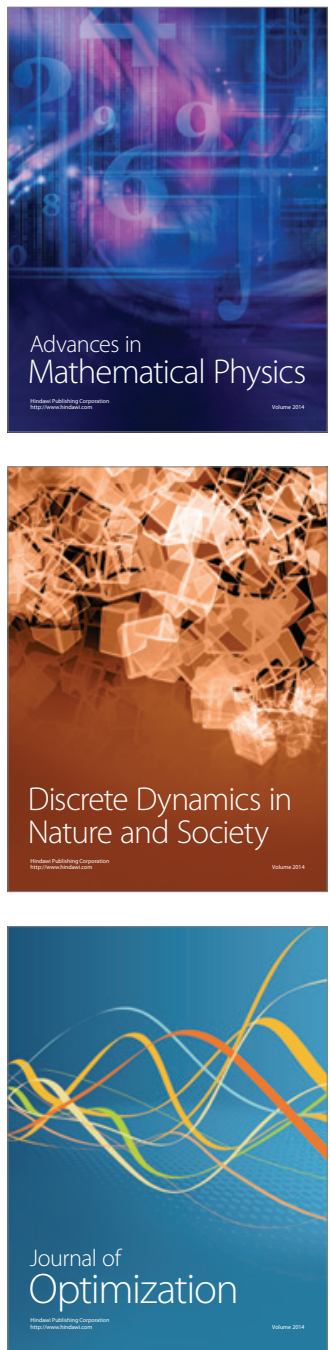